\title{
The Exact Distribution of the TSLS Estimator for a Non- Gaussian Just-Identified Linear Structural Equation
}

by

Giovanni Forchini

August 30, 2006

\begin{abstract}
Recent interest in the exact distribution of the TSLS estimator of the coefficients of a linear structural equation has focused on the case where the errors are independent normal random variables. This raises the issue of how representative these results are of cases where the error distribution is not normal. This paper shows that in a just-identified structural equation with one endogenous variable the structure of the exact results does not change if we allow the vector of the errors to be in the family of elliptical distributions.
\end{abstract}

\section{Introduction}

The exact distribution of the two-stage least square (TSLS) estimator of the coefficients of the endogenous variables in a linear structural equation is known for the case where the errors are independently normally distributed (e.g. Phillips (1983), and Woglom (2001)). No exact results are available for more general error structures.

In this note, we study the exact distribution of the TSLS estimator without the assumption of normality, and investigate how existing exact results generalize. Following Nelson and Startz (1990), Maddala and Jeong (1992) and Woglom (2001), we focus on a structural equation that is just-identified and has only one endogenous variable. In this simple case, the TSLS estimator of the coefficient of the endogenous variable has the form of a ratio of two correlated random variables. Under the assumption of Gaussian errors, these are jointly normal. Results for ratios of non-normal distributions are 
available but are scattered in the statistics literature. Kotlarski (1964) and Arnold and Brockett (1992) have studied the distribution of ratios of two random variables, $(X, Y)$ say, in the family of spherical and elliptical distributions, respectively, and have found that if $(X, Y)$ has zero mean, then $X / Y$ has a Cauchy distribution. More recently, Nadarajah (2006) has investigated the exact probability function of a ratio of two random variables of non-zero mean having jointly the elliptical Pearson-type II and -type VII, and Kotz-type distributions.

In this paper, we generalize known results from exact distribution theory as well as existing results for the ratio of two random variables. We consider the case where the TSLS estimator can be written as a ratio of two elliptically distributed random variables, and derive its density in terms of quantities having simple statistical interpretation. We give a very elementary proof of the fact that if the structural equation is unidentified, then the TSLS estimator has a Cauchy distribution. If the structural equation is identified, the density of the TSLS estimator is more complicated but its structure is the same for all possible distributions of the numerator and the denominator in the elliptical family. As for the normal case, no integer moments of the TSLS estimator exist.

The rest of this note is organized as follows. Section 2 gives some preliminary results and establishes the notation used in Section 3 to study the ratio of two random variables in the family of elliptical distributions. Section 4 concludes.

\section{The model, preliminary results and notation}

Consider a just-identified linear structural equation with one endogenous variable

$$
\begin{gathered}
y=Y \beta+u \\
Y=Z \Pi+V
\end{gathered}
$$

where $y$ and $Y$ denote the $(T \times 1)$ vectors of endogenous variables, $Z$ denotes a $(T \times 1)$ fixed vector of exogenous variables, and $u$ and $V$ are random vectors. Insert (2) into (1) to obtain a reduced form for $y$

$$
y=Z \Pi \beta+v,
$$

where $v=u+V \beta$. Let 


$$
(\hat{\pi}, \hat{\Pi})=\left(Z^{\prime} Z\right)^{-1} Z^{\prime}(y, Y) .
$$

The TSLS estimator of $\beta$ is

$$
\hat{\beta}=\hat{\pi} / \hat{\Pi} .
$$

For this simple model, the TSLS estimator and the limited information maximum likelihood estimator coincide. Clearly, if the errors are normally distributed, $(\hat{\pi}, \hat{\Pi})$ are jointly normal random variables, and the exact properties of $\hat{\beta}$ have been studied by Phillips and Wickens (1978) (Solution 6.19, pp. 351-355), Phillips (1983), Nelson and Startz (1990), Maddala and Jeong (1992), Woglom (2001) and Hillier (2006). In this note, we study the case where $(\hat{\pi}, \hat{\Pi})$ has an arbitrary elliptical distribution, and derive the density of the ratio $\hat{\pi} / \hat{\Pi}$ in terms of quantities having statistical interpretation. The fact that $(\hat{\pi}, \hat{\Pi})$ belongs to the elliptical family is implied by the fact that $(v, V)$ is also elliptical, since $(\hat{\pi}, \hat{\Pi})$ is an affine transformation of $(v, V)$. By focussing on the distribution of $(\hat{\pi}, \hat{\Pi})$ we can also look at cases where the vector of instruments is random (rather than fixed): if $(v, V) \mid Z \sim N\left(0, I_{T} \otimes \Omega\right)$ and $Z$ has any distribution, then $(\hat{\pi}, \hat{\Pi}) \mid Z \sim N\left((\Pi \beta, \Pi),\left(Z^{\prime} Z\right)^{-1} \otimes \Omega\right)$, and the marginal distribution of $(\hat{\pi}, \hat{\Pi})$ is a covariance-matrix-mixed-normal and is in the elliptical family.

We suppose that the joint density of the random variables $(\hat{\pi}, \hat{\Pi})$ has the form

$$
\operatorname{pdf}(\hat{\pi}, \hat{\Pi})=c_{2}|\Omega|^{-1 / 2} \phi\left(\left(\begin{array}{l}
\hat{\pi}-\Pi \beta \\
\hat{\Pi}-\Pi
\end{array}\right) \Omega^{-1}\left(\begin{array}{l}
\hat{\pi}-\Pi \beta \\
\hat{\Pi}-\Pi
\end{array}\right)\right)
$$

for some function $\phi$, and $-\infty<\hat{\pi}<\infty,-\infty<\hat{\Pi}<\infty$, where $\Omega$ is a $(2 \times 2)$ positive definite matrix

$$
\Omega=\left(\begin{array}{ll}
\omega_{11} & \omega_{21} \\
\omega_{21} & \omega_{22}
\end{array}\right)
$$


and $c_{2}$ is a normalizing constant indexed by the dimension of the random vector $(\hat{\pi}, \hat{\Pi})$ (cf. Definition 1.5.2 of Muirhead (1982)). If they exist, the mean of $(\hat{\pi}, \hat{\Pi})$ is $(\Pi \beta, \Pi)$, and the covariance matrix is $\alpha \Omega$ where $\alpha$ is a scalar constant depending on the characteristic function of $(\hat{\pi}, \hat{\Pi})$. No other assumptions are used.

Let

$$
\left(\begin{array}{l}
w_{1} \\
w_{2}
\end{array}\right)=\Omega^{-1 / 2}\left(\begin{array}{l}
\hat{\pi}-\Pi \beta \\
\hat{\Pi}-\Pi
\end{array}\right)
$$

where $\Omega^{-1 / 2}$ denotes the inverse of the matrix square root of $\Omega$ (the Jacobian of the transformation is $\left.\operatorname{det}(\Omega)^{1 / 2}\right)$. Then, the density of $\left(w_{1}, w_{2}\right)$ is

$$
p d f\left(w_{1}, w_{2}\right)=c_{2} \phi\left(w_{1}^{2}+w_{2}^{2}\right) .
$$

We use (9) to define two functions that will be relevant later on. Firstly, the density function of $t=w_{1}^{2}+w_{2}^{2}$ is

$$
p d f(t)=\pi c_{2} \phi(t) .
$$

In the rest of this note, $\Phi(t)=\pi c_{2} \int_{0<q<t} \phi(q) d q$ denotes the cumulative distribution function of $t$. Secondly, from (9) we can obtain the joint density of $t_{1}=w_{1}^{2}$ and $w_{2}$ as $p d f\left(t_{1}, w_{2}\right)=c_{2} \phi\left(t_{1}+w_{2}^{2}\right) t_{1}^{-1 / 2}$, and integrating out $t_{1}$ we obtain the marginal density of $w_{2}$

$$
p d f\left(w_{2}\right)=c_{2} \int_{t_{1}>0} \phi\left(t_{1}+w_{2}^{2}\right) t_{1}^{-1 / 2} d t_{1}=c_{2} \phi_{m}\left(w_{2}^{2}\right) .
$$

The marginal densities of $w_{1}$ and $w_{2}$ have the same functional form. We now define the incomplete marginal density of $w_{2}$ as

$$
\phi_{w_{2}^{2}}\left(t_{1}\right)=c_{2} \int_{0<q<t_{1}} \phi\left(q+w_{2}^{2}\right) q^{-1 / 2} d q .
$$

The conditional density of $t_{1}$ given $w_{2}$ is $p d f\left(t_{1} \mid w_{2}^{2}\right)=\phi\left(t_{1}+w_{2}^{2}\right) t_{1}^{-1 / 2} / \phi_{m}\left(w_{2}^{2}\right)$ and the conditional cumulative distribution function of $t_{1}$ given $w_{2}$ is 
$\Phi_{c}\left(t_{1} \mid w_{2}^{2}\right)=\int_{0<q<t_{1}} p d f\left(t_{1} \mid w_{2}^{2}\right) d q$, therefore we have ${ }^{1} \phi_{w_{2}^{2}}\left(t_{1}\right)=\Phi_{c}\left(t_{1} \mid w_{2}^{2}\right) \times c_{2} \phi_{m}\left(w_{2}^{2}\right)$.

Both functions $\phi_{w_{2}^{2}}\left(t_{1}\right)$ and $\Phi(t)$ can be efficiently evaluated by numerical integration, and, often, also analytically using computer packages such as Mathematica or Maple. This is certainly the case for the examples considered by Nadarajah (2006).

\section{Main results}

Let $\hat{\pi}=\hat{\Pi} \hat{\beta}$ in equation (6) (the Jacobian is $|\hat{\Pi}|$ ) so that the density of $\hat{\beta}$ can be written after simplification as

$$
p d f(\hat{\beta})=c_{2} \operatorname{det}(\Omega)^{-1 / 2} \int_{-\infty<\hat{\Pi}<+\infty}|\hat{\Pi}| \phi\left(a(\hat{\beta})(\hat{\Pi}-b(\hat{\beta}) / a(\hat{\beta}))^{2}+c-b(\hat{\beta})^{2} / a(\hat{\beta})\right) d \hat{\Pi}
$$

where

$$
\begin{aligned}
a(\hat{\beta}) & =\left(1 / \omega_{22}\right)\left(1+\left(\omega_{22} / \omega_{11.2}\right)\left(\hat{\beta}-\omega_{21} / \omega_{22}\right)^{2}\right) \\
b(\hat{\beta}) & =\left(\Pi / \omega_{22}\right)\left(1+\left(\omega_{22} / \omega_{11.2}\right)\left(\hat{\beta}-\omega_{21} / \omega_{22}\right)\left(\beta-\omega_{21} / \omega_{22}\right)\right) \\
c & =\left(\Pi^{2} / \omega_{22}\right)\left(1+\left(\omega_{22} / \omega_{11.2}\right)\left(\beta-\omega_{21} / \omega_{22}\right)^{2}\right)
\end{aligned}
$$

and by the Cauchy-Schwartz inequality $b(\hat{\beta})^{2} \leq c \times a(\hat{\beta})$. In the display above we have set $\omega_{11.2}=\omega_{11}-\omega_{21}^{2} / \omega_{22}$. The quantity $\omega_{21} / \omega_{22}$ is the probability limit of the ordinary least squares estimator of $\beta$ in the structural equation (1).

Before dealing with the general case we focus on the totally unidentified case where $\Pi=0$. In this case, equation (12) simplifies to

$$
p d f(\hat{\beta})=c_{2} \operatorname{det}(\Omega)^{-1 / 2} \int_{-\infty<\hat{\Pi}<+\infty}|\hat{\Pi}| \phi\left(a(\hat{\beta}) \hat{\Pi}^{2}\right) d \hat{\Pi}
$$

and after a simple change of variable, integration yields our first result.

Proposition 1. If $\Pi=0$, the density of $\hat{\beta}$ is

$$
\operatorname{pdf}(\hat{\beta})=\pi^{-1} \operatorname{det}(\Omega)^{-1 / 2} a(\hat{\beta})^{-1} \text {. }
$$

1 A document available at http://www-personal.buseco.monash.edu.au/ forchini/ contains detailed derivations of this result as well as those of Propositions 1 and 2 below. 
If the mean of $(\hat{\pi}, \hat{\Pi})$ is zero, then $\hat{\beta}$ has a Cauchy distribution with location parameter $\omega_{21} / \omega_{22}$, the probability limit of the ordinary least squares estimator of $\beta$ in the structural equation, and scale parameter $\omega_{22} / \omega_{11.2}$. Both the location and the scale of the Cauchy distribution depend on $\Omega$ only. We will see shortly that the specific function $\phi$ becomes relevant when $\Pi \neq 0$. Proposition 1 depends on the assumption that $(\hat{\pi}, \hat{\Pi})$ has an elliptical distribution. In general, there are distributions of $(\hat{\pi}, \hat{\Pi})$ for which $\hat{\beta}=\hat{\pi} / \hat{\Pi}$ does not have a Cauchy distribution (cf. Kotlarski (1964)). For example, if we assume that $(\hat{\pi}, \hat{\Pi})$ are independent chi-square random variables, their ratio would be proportional to an F distribution.

In the general case, we can let $\hat{\Pi}=a(\hat{\beta})^{-1 / 2}\left(s+b(\hat{\beta}) a(\hat{\beta})^{-1 / 2}\right)$ so that

$$
p d f(\hat{\beta})=c_{2} \operatorname{det}(\Omega)^{-1 / 2} a(\hat{\beta})^{-1} \int_{-\infty<s<+\infty}\left|s+b(\hat{\beta}) a(\hat{\beta})^{-1 / 2}\right| \phi\left(s^{2}+c-b(\hat{\beta})^{2} / a(\hat{\beta})\right) d s .
$$

Writing $s$ in polar coordinates as $s=h t^{1 / 2}$ where $h^{2}=1$ and $t>0$ (the Jacobian is $(1 / 2) t^{-1 / 2}$ ) we have:

$$
\begin{gathered}
p d f(\hat{\beta})=c_{2} \operatorname{det}(\Omega)^{-1 / 2} a(\hat{\beta})^{-1} \int_{0<<<+\infty} \phi\left(t+c-b(\hat{\beta})^{2} / a(\hat{\beta})\right) t^{-1 / 2} \\
(1 / 2) \int_{h^{2}=1}\left|h t^{1 / 2}+b(\hat{\beta}) a(\hat{\beta})^{-1 / 2}\right|(d h) d t .
\end{gathered}
$$

The integral over $h^{2}=1$ can be evaluated explicitly as

$$
(1 / 2) \int_{h^{2}=1}\left|h t^{1 / 2}+b(\hat{\beta}) a(\hat{\beta})^{-1 / 2}\right|(d h)=\left\{\begin{array}{cc}
t^{1 / 2} & \text { if } t>b(\hat{\beta})^{2} / a(\hat{\beta}) \\
|b(\hat{\beta})| a(\hat{\beta})^{-1 / 2} & \text { if } 0<t<b(\hat{\beta})^{2} / a(\hat{\beta})
\end{array}\right.
$$

and inserting this into equation (14) we obtain

$$
\begin{aligned}
p d f(\hat{\beta}) & =c_{2} \operatorname{det}(\Omega)^{-1 / 2}|b(\hat{\beta})| a(\hat{\beta})^{-3 / 2} \int_{0<t<b(\hat{\beta})^{2} / a(\hat{\beta})} \phi\left(t+c-b(\hat{\beta})^{2} / a(\hat{\beta})\right) t^{-1 / 2} d t \\
& +c_{2} \operatorname{det}(\Omega)^{-1 / 2} a(\hat{\beta})^{-1} \int_{b(\hat{\beta})^{2} / a(\hat{\beta})<t<+\infty} \phi\left(t+c-b(\hat{\beta})^{2} / a(\hat{\beta})\right) d t .
\end{aligned}
$$


We note that

$$
c_{2} \int_{0<t<b(\hat{\beta})^{2} / a(\hat{\beta})} \phi\left(t+c-b(\hat{\beta})^{2} / a(\hat{\beta})\right) t^{-1 / 2} d t=\phi_{c-b(\hat{\beta})^{2} / a(\hat{\beta})}\left(b(\hat{\beta})^{2} / a(\hat{\beta})\right)
$$

and, after defining $z=t+c-b(\hat{\beta})^{2} / a(\hat{\beta})$, the second integral in (15) becomes

$$
\pi c_{2} \int_{c<z<+\infty} \phi(z) d z=1-\Phi(c) \text {. }
$$

Therefore, we have our second result.

Proposition 2. The density of $\hat{\beta}$ is

$$
\begin{aligned}
\operatorname{pdf}(\hat{\beta}) & =\operatorname{det}(\Omega)^{-1 / 2}|b(\hat{\beta})| a(\hat{\beta})^{-3 / 2} \phi_{c-b(\hat{\beta})^{2} / a(\hat{\beta})}\left(b(\hat{\beta})^{2} / a(\hat{\beta})\right) \\
& +\pi^{-1} \operatorname{det}(\Omega)^{-1 / 2} a(\hat{\beta})^{-1}[1-\Phi(c)]
\end{aligned}
$$

When $\Pi=0$ Proposition 2 reduces to Proposition 1 . When $\Pi \neq 0$, then the density of $\hat{\beta}$ is the sum of two components depending on $\Phi(c)$ and the incomplete marginal density for $w_{2}$ previously defined $\phi_{c-b(\hat{\beta})^{2} / a(\hat{\beta})}\left(b(\hat{\beta})^{2} / a(\hat{\beta})\right)$. Notice that the latter can be written as the product $\Phi_{c}\left(t_{1} \mid w_{2}^{2}\right) \times c_{2} \phi_{m}\left(w_{2}^{2}\right)$ evaluated at $t_{1}=b(\hat{\beta})^{2} / a(\hat{\beta})$ and $w_{2}^{2}=1-t_{1}$.

The component in the second line of equation (16) is symmetric and has only one mode at $\omega_{21} / \omega_{22}$, moreover its relative importance in determining the shape of the density of $\hat{\beta}$ decreases very quickly as $c$ becomes large. The component in the first line of equation (16) is not symmetric. Since this term must integrate to $\Phi(c)$, its tails must go to zero as $|\hat{\beta}|$ increases if $p d f(\hat{\pi}, \hat{\Pi})$ is defined on the whole two dimensional space. Moreover, this term has a minimum of zero when $b(\hat{\beta})=0$ (and $\Pi \neq 0$ ) at $\hat{\beta}=\left(\omega_{11}-\beta \omega_{21}\right) /\left(\omega_{21}-\beta \omega_{22}\right)$, so that the first line of equation (16) must have (at least) two modes, but one of them could be very small. The potential bimodality of $\hat{\beta}$ has been studied by Nelson and Startz (1990), Maddala and Jeong (1992) and Woglom (2001) in the context of Gaussian just-identified structural equations. Forchini (2006) gives a detailed discussion of the possible bimodality in just- and over-identified structural 
equations with Gaussian errors.

In the case where $(\hat{\pi}, \hat{\Pi})$ are jointly normal we have $\phi(t)=\exp \{-t / 2\}$. If for the sake of simplicity we assume that $\Omega=I_{2}$, then we can easily find that

$$
\Phi(t)=\pi \int_{0<q<t}(2 \pi)^{-1} \exp \{-q / 2\} d q=1-\exp \{-t / 2\}
$$

and this is the cumulative distribution function of a random variable having a chi-squared distribution with two degrees of freedom. Analogously we find that

$$
\phi_{w_{2}^{2}}(t)=\frac{\exp \left\{-w_{2}^{2} / 2\right\}}{\sqrt{2 \pi}} \int_{0<q<t} \frac{\exp \{-q / 2\} q^{-1 / 2}}{\sqrt{2 \pi}} d q
$$

and the remaining integral is the cumulative distribution function of a random variable having a chi-squared distribution with one degree of freedom. It can also be evaluated explicitly as

$$
\phi_{w_{2}^{2}}(t)=\exp \left\{-w_{2}^{2} / 2\right\} t^{1 / 2} F_{1}(1 / 2 ; 3 / 2 ;-t / 2) / \pi
$$

It is clear from equations (17) and (18) that both $\phi_{c-b(\hat{\beta})^{2} / a(\hat{\beta})}\left(b(\hat{\beta})^{2} / a(\hat{\beta})\right)$ and $\Phi(c)$ are incomplete gamma functions. If $(\hat{\pi}, \hat{\Pi})$ belongs to a bivariate Pearson-type II distribution, then the quantity above would be incomplete beta functions. In the general case they are the incomplete functions based on $\phi$, but in practice the function $\phi$ has a simple functional form so that $\phi_{c-b(\hat{\beta})^{2} / a(\hat{\beta})}\left(b(\hat{\beta})^{2} / a(\hat{\beta})\right)$ and $\Phi(c)$ can be written in terms of hypergeometric functions, or infinite sums of hypergeometric functions.

Returning to the general case, we note that the second line of the density of $\hat{\beta}$ in equation (16) depends on $a(\hat{\beta})^{-1}$ only. Thus

Proposition 3. The TSLS estimator $\hat{\beta}=\hat{\pi} / \hat{\Pi}$ does not have integer moments.

This implies that the TSLS estimator has a distribution with thick tails so that the chance of observing extreme values is relatively large. Clearly, caution needs to be exercised when studying the properties of $\hat{\beta}$ using Monte Carlo simulations. 


\section{Conclusions}

This paper has studied the exact distribution of the TSLS estimator of $\beta$ in a justidentified structural equation with one endogenous variable where $(\hat{\pi}, \hat{\Pi})$ in the elliptical family of distributions. This is a very general family of distributions and contains most of the distributions considered in practice. We suggested an elementary proof that if the structural equation is unidentified then the TSLS estimator has a Cauchy distribution. If the structural equation is identified, the exact distribution of the TSLS estimator is more complicated and depends on the incomplete functions $\phi_{w_{2}^{2}}\left(t_{1}\right)$ and $\Phi(t)$ which are integrals of the underlying function $\phi$ defining the joint density of $(\hat{\pi}, \hat{\Pi})$. As for the normal case, no integer moments exist, and bimodality may arise depending on the values of some key parameters.

\section{References}

Arnold, B. C. and P. L. Brockett (1992). "On Distributions Whose Component Ratios are Cauchy." The American Statistician 46: 25-26.

Forchini, G. (2006). "On the Bimodality of the Exact Distribution of the TSLS Estimator." Econometric Theory Forthcoming.

Hillier, G. H. (2006). "Yet More on The Exact Properties of IV Estimators." Econometric Theory Forthcoming.

Kotlarski, I. (1964). "On Bivariate Random Variables Where the Quotient of their Coordinates Follows some Known Distribution." The Annals of Mathematical Statistics 35: 1673-1684.

Maddala, G. S. and J. Jeong (1992). "On the Exact Small Sample Distribution of the Instrumental Variable Estimator." Econometrica 60: 181-183.

Muirhead, R. J. (1982). Aspects of Multivariate Statistical Theory. New York, John Wiley \& Sons.

Nadarajah, S. (2006). "On the Ratio X/Y for some Elliptically Symmetric Distributions." Journal of Multivariate Analysis 97: 342-358.

Nelson, C. R. and R. Startz (1990). "Some Further Results on the Small Sample Properties of the Instrumental Variable Estimator." Econometrica 58: 967-976.

Phillips, P. C. B. (1983). "Exact Small Sample Theory in the Simultaneous Equations Model". Handbook of Econometrics, chap. 8. Z. Griliches and M. D. Intriligator. Amsterdam, North-Holland. I: 449-516.

Phillips, P. C. B. and M. R. Wickens (1978). Exercises in Econometrics, Volume II, Ballinger and Philip Allan.

Woglom, G. (2001). "More Results on the Exact Small Sample Properties of the Instrumental Variable Estimator." Econometrica 69: 1381-1389. 